3. В. Самсония, И. Г. Самхарадзе (Ин-т вычислит. математики АН Грузии, Тбилиси)

\title{
О КВАЗИКОНФОРМНЫХ ОТОБРАЖЕНИЯХ, СООТВЕТСТВУЮЩИХ УРАВНЕНИЮ БЕЛЬТРАМИ
}

By using methods of integral equations, we investigate problems of conformal and quasiconformal mappings of close domains.

Досліцжуються задачі конформиого та квазіконформиого відображень близьких областен 3 допомогою методів іптегральних рівнянь.

В данной работе усиливаются результаты, полученные авторами ранее (см. замечание в конце статьи), и приводятся их некоторые применения. Следует также отметитъ, что в квазиконформном случае отображения близких областей, по-видимому, изучаются впервые.

1. Метод интегральных уравнений. Глобальный гомеоморфизм $\tilde{W}(z)$ всей комплексной плоскости $E$ уравнения Бельтрами

$$
W_{\bar{z}}=q(z) W_{z}
$$

в предположении, что $q(z) \in L_{p}(E), p>2,|q(z)| \leq Q_{0}<1$, можно строить по схеме, предложенной в [1], с помощью интегральных операторов

$$
T_{G} f=-\frac{1}{\pi} \iint_{G} \frac{f(\zeta)}{\zeta-z} d \xi d \eta, \quad \Pi_{G} f=-\frac{1}{\pi} \iint_{G} \frac{f(\zeta)}{(\zeta-z)^{2}} d \xi d \eta
$$

где $G$ - конечная область плоскости $E$. В дальнейшем будем предполагать $q(z)$ достаточно гладкой функцией в $\bar{G}$, а вне $\bar{G} q(z) \equiv 0$.

Согласно [1] (гл. 2), функция

$$
\tilde{W}(z)=z+T_{G} f .
$$

где $f(z)$ - единственное решение плоского сингулярного уравнения

$$
f(z)-q(z) \Pi_{G} f=q(z),
$$

осуществляет гомеоморфное отображение плоскости $E$ на себя и $\tilde{W}(\infty)=\infty$, $z^{-1} \bar{W}(z) \rightarrow 1$ при $|z| \rightarrow \infty$. Такая функция единственна.

Если $q(z) \in C_{\gamma}(\bar{G})$ и $\partial G \in C_{\alpha}^{1}, 0<\alpha<1$, где $C_{\alpha}^{1}$ - класс контуров Ляпунова, то, согласно [2], $\tilde{W}_{z}, \tilde{W}_{\bar{z}} \in C_{\gamma_{0}}$, где $0<\gamma_{0} \leq \min \{\alpha, \gamma\}$. В случае $f(z) \in C_{1}(\bar{G})$ для $\Pi_{G} f$ справедливо представленне [1]

$$
\Pi_{G} f=T_{G}\left(\frac{\partial f}{\partial z}\right)+\frac{1}{2 \pi i} \int_{\partial G} \frac{f(\zeta) d \zeta}{\zeta^{2}(\zeta-z)} .
$$

Гомеоморфизм $\bar{W}(z)$ известен под названием основного гомеоморфизма уравнения (1).

Пусть коэффициент $q(z)$ уравнения Бельтрами задан в некоторой конечной односвязной области $G_{0}$ и прннадлежит классу $C_{\gamma}\left(\bar{G}_{0}\right), 0<\gamma<1,0 \in G_{0}$.

Рассмотрим на комплексной плоскости односвязную область $G, G \subseteq G_{0}$, $0 \in G$, ограниченную контуром $\Gamma=\partial G, \Gamma \in C_{\alpha}^{l}, 0<\alpha<1$. Имея в виду свойства решений уравнения Бельтрами, функщию $W=W(z), W(0)=0$, $W\left(z_{1}\right)=1, z_{1} \in \Gamma$, реализуюшую квазиконформное отображение области $G$ на единичный круг, будем искать в виде 


$$
W=[\tilde{W}(z)-\tilde{W}(0)] e^{\psi(z)}=[\tilde{W}(z)-\tilde{W}(0)] \exp \left(\frac{1}{\pi i} \int_{\Gamma} \frac{v(t) d \tilde{W}}{\tilde{W}(t)-\tilde{W}(z)}+i C\right),
$$

где $d \tilde{W}=\tilde{W}_{t} d t+\tilde{W}_{\bar{i}} d \bar{t}, \quad \tilde{W}(z)-$ основной гомеоморфизм уравнения (1) $\left(q(z) \in 0\right.$ вне $\left.\bar{G}_{0}, \partial G_{0} \in C_{\alpha}^{\prime}\right)$. Действительная константа $C$ и действительная фунхция $v(t)$ определяются исходя из требовании, предъявленных $\mathrm{K}$ $W(z)$.

Тахим образом прнходим к справедливости следующего предложения [3]:

функция $W=W(z)$, заданная в $G$ формулой (5), удовлетворяет уравнению (1) и однолистно отображает $G$ на единичның круг с выполнением условий $W(0)=0, W\left(z_{1}\right)=1$, если $v(t)$ является решением интегрального уравнения

$$
v(t)+\frac{1}{\pi} \int_{\Gamma} v(\tau) \operatorname{darg}[\tilde{W}(\tau)-\tilde{W}(t)]=-\ln |\tilde{W}(t)-\tilde{W}(0)|
$$

и

$$
C=-\arg \left[\tilde{W}\left(z_{1}\right)-\tilde{W}(0)\right]-\frac{1}{\pi} \int_{\Gamma} v(\tau) d \ln \left|\tilde{W}(\tau)-\tilde{W}\left(z_{1}\right)\right| .
$$

Уравнение (6) имеет единственное решение $v(t)$, принадлежащее некоторому классу Гељьера.

В конформном случае $\tilde{W}(z)=z$ и, если

$$
\Psi(z)=\frac{1}{\pi i} \int_{\Gamma} \frac{v(t) d t}{t-z}+\frac{i}{\pi} \int_{\Gamma} \frac{v(t)}{\rho} d \rho \quad(\rho=|t|),
$$

приходим к конструкцин, предложенной в [4].

В предлагаемой работе метод плоских и контурных интегральных уравнений применяется в качестве конструктивного аппарата в задачах отображения близких областей. В приведенной ниже постановке этот метод можно применить как в конформном, так и в квазиконформном случаях.

2. О задаче конформного отображения близких областей. ПредполоЖим, что уравнение границы $\Gamma$ задано в параметрической форме

$$
t=g(\tau), \quad 0 \leq \tau \leq 2 \pi ; \quad g(0)=g(2 \pi) .
$$

Рассмотрим другую односвязную область $\tilde{G}$ типа $G$ на комплексной плоскости с уравнением границы $\tilde{\Gamma}$.

$$
t=\tilde{g}(\tau), \quad 0 \leq \tau \leq 2 \pi ; \quad \tilde{g}(0)=\tilde{g}(2 \pi) .
$$

Определение. Область $\tilde{G}$ назовел $\varepsilon$-близкой $(\varepsilon>0) \kappa$ области $G$, если өыполняются условия

$$
|g(\tau)-\tilde{g}(\tau)| \leq \varepsilon, \quad\left\|g^{\prime}(\tau)-\tilde{g}^{\prime}(\tau)\right\|_{C_{\alpha}} \leq \varepsilon .
$$

Для лобого $\varepsilon>0$, разумеется, образуется бесконечное множество областеи $\varepsilon$-близких к $G$. Такое множество обозначим через $G_{\varepsilon}$.

Интегральное уравнение (6) представим в комплексном виде

$$
v\left(t_{0}\right)+\frac{1}{2 \pi i} \int_{\Gamma} \frac{1}{t^{\prime}} K\left(t ; t_{0}\right) v(t) d t=-\ln \left|t_{0}\right|, \quad t_{0} \in \Gamma .
$$

где 


$$
\begin{gathered}
K\left(t ; t_{0}\right)=\frac{t^{\prime}-t_{0}^{\prime}}{t-t_{0}}+\frac{\bar{t}^{\prime}-\bar{t}_{0}^{\prime}}{\bar{t}-\bar{t}_{0}}+\frac{t_{0}^{\prime} \frac{\bar{t}-\bar{t}_{0}}{t-t_{0}}-\bar{t}_{0}^{\prime}}{\bar{t}-\bar{t}_{0}}, \\
t^{\prime}=\frac{d g(\tau)}{d \tau}, \quad t_{0}^{\prime}=\left(\frac{d g(\tau)}{d \tau}\right)_{\tau=\tau_{0}}, \quad \tau_{0} \in[0,2 \pi] .
\end{gathered}
$$

Аналогично, интегральное уравнение для области $\bar{G}$ имеет вид

$$
\tilde{v}\left(t_{0}\right)+\frac{1}{2 \pi i} \int_{\tilde{\Gamma}} \frac{1}{t^{\prime}} \tilde{K}\left(t ; t_{0}\right) \tilde{v}(t) d t=-\ln \left|t_{0}\right|, \quad t_{0} \in \tilde{\Gamma} .
$$

где $\tilde{K}\left(t ; t_{0}\right)$ получается из $K\left(t ; t_{0}\right)$ в предположении, что $t=\tilde{g}(\tau), t_{0}=\tilde{g}\left(\tau_{0}\right)$. Справедлива следуюшая теорема.

Теорема 1. Если границы областей $G$ и $\tilde{G} \in G_{\varepsilon}, 0<\varepsilon \leq \varepsilon_{0}$, принадлежат классу $C_{\alpha}^{\prime}, 0<\alpha<1$, то справедливо неравенство

$$
\|v(\tau)-\tilde{v}(\tau)\|_{C_{\alpha-\beta}}<A_{0}(\beta)\|v\|_{C_{\alpha-\beta}} \varepsilon,
$$

где $v(t)$ и $\tilde{v}(\tau)$ - единственные решения интегральных уравнений (9) и (9') соответственно. Константа $A_{0}(\beta)$ и малое число $\varepsilon_{0}$ определяются зада. ниель исходной области $G ; \beta$-любое положительное число, льеньее $\alpha$.

Доказательстео. Используя параметр $\tau$, уравнения $(9)$ и $\left(9^{\prime}\right)$ представим в виде

$$
\begin{aligned}
& v\left(\tau_{0}\right)+\frac{1}{2 \pi i} \int_{0}^{2 \pi} \frac{S\left(\tau_{0} ; \tau\right) M\left(\tau ; \tau_{0}\right)}{\left|e^{i \tau}-e^{i \tau_{0}}\right|^{\beta}}\left(\operatorname{ctg} \frac{\tau-\tau_{0}}{2}+i\right)\left|e^{i \tau}-e^{i \tau_{0}}\right|^{\beta} v(\tau) d \tau=-\ln \left|g\left(\tau_{0}\right)\right|, \\
& \tilde{v}\left(\tau_{0}\right)+\frac{1}{2 \pi i} \int_{0}^{2 \pi} \frac{\tilde{S}\left(\tau_{0} ; \tau\right) \tilde{M}\left(\tau ; \tau_{0}\right)}{\left|e^{i \tau}-e^{i \tau_{0}}\right|^{\beta}}\left(\operatorname{ctg} \frac{\tau-\tau_{0}}{2}+i\right)\left|e^{i \tau}-e^{i \tau_{0}}\right|^{\beta} \tilde{v}(\tau) d \tau=-\ln \left|\tilde{g}\left(\tau_{0}\right)\right|,
\end{aligned}
$$

где $v(\tau)=v[g(\tau)], \quad \vec{v}(\tau)=\tilde{v}[\tilde{g}(\tau)]$.

Кроме того, введены следующие обозначення:

$$
\frac{d t}{t-t_{0}}=\frac{g^{\prime}(\tau) d \tau}{g(\tau)-g\left(\tau_{0}\right)}=M\left(\tau_{0} ; \tau\right)\left(\operatorname{ctg} \frac{\tau-\tau_{0}}{2}+i\right) d \tau,
$$

где функция

$$
M\left(\tau_{0} ; \tau\right)=\frac{g^{\prime}(\tau)}{2 i e^{i \tau}} \frac{e^{i \tau}-e^{i \tau_{0}}}{g(\tau)-g\left(\tau_{0}\right)}
$$

удовлетворяет условию Гельдера с показателем $\alpha$ относительно своих аргументов, т. е. принадлежит классу $C_{\alpha}(\Gamma \times \Gamma)$;

$$
\begin{gathered}
S\left(\tau_{0} ; \tau\right)=\frac{g(\tau)-g\left(\tau_{0}\right)}{g^{\prime}(\tau)} K\left[g(\tau) ; g\left(\tau_{0}\right)\right], \\
S\left(\tau_{0} ; \tau\right) \in C_{\alpha}(\Gamma \times \Gamma), \quad S\left(\tau_{0} ; \tau_{0}\right)=0,
\end{gathered}
$$

$\beta$ - любое число из $(0, \alpha)$. Подобно определяются значения для выражений $\tilde{M}\left(\tau_{0} ; \tau\right), \tilde{S}\left(\tau_{0} ; \tau\right)$.

Представим интегральные уравнения (11) и (11) в операторном виде 


$$
\begin{gathered}
A v=v+H v=f_{0}, \\
\tilde{A} \tilde{v}=\bar{v}+\tilde{H} \tilde{v}=\tilde{f}_{0},
\end{gathered}
$$

где $f_{0}=-\ln \left|g\left(\tau_{0}\right)\right|, \tilde{f}_{0}=-\ln \left|\tilde{g}\left(\tau_{0}\right)\right|, A=I+H, \tilde{A}=I+\tilde{H}$, а $H$ и и $\tilde{H} \tilde{v}-$ интегральные операторы, входящие в левые части уравнений (11) и (11') соответственно.

Рассмотрим выражение

$$
(H-\tilde{H}) \nu\left(\tau_{0}\right)=\frac{1}{2 \pi i} \int_{0}^{2 \pi} \tilde{K}^{*}\left(\tau_{0} ; \tau\right)\left(\operatorname{ctg} \frac{\tau-\tau_{0}}{2}+i\right)\left|e^{i \tau}-e^{i \tau_{0}}\right|^{\beta} v(\tau) d \tau,
$$

где

$$
\tilde{K}^{*}\left(\tau_{0} ; \tau\right)=\frac{S\left(\tau_{0} ; \tau\right) M\left(\tau_{0} ; \tau\right)-\tilde{S}\left(\tau_{0} ; \tau\right) \tilde{M}\left(\tau_{0} ; \tau\right)}{\left|e^{i \tau}-e^{i \tau_{0}}\right|^{\beta}} .
$$

Воспользуемся следуюшими результатами из [5]:

a) функция двух переменных

$$
\psi\left(t ; t_{0}\right)=\frac{\varphi(t)-\varphi\left(t_{0}\right)}{\left|t-\dddot{t}_{0}\right|^{\lambda}}, \quad\left(t ; t_{0} \in \Gamma\right)
$$

удовлетворяет на $\Gamma$ условию Гельдера с показателем $\mu_{0}-\lambda$, если $\varphi(t) \epsilon$ $\epsilon C_{\mu_{0}}(\Gamma)$ и $0 \leq \lambda<\mu_{0}<1$; более того, справедлива оценка

$$
\left\|\psi\left(t ; t_{0}\right)\right\|_{C_{\mu_{0}-\lambda}} \leq A^{*}(1+\lambda)
$$

где $A^{*} \geq \frac{\left|\varphi(t)-\varphi\left(t_{0}\right)\right|}{\left|t-t_{0}\right|^{\lambda}}$ (см. $\S 5$ и 6 указанной работы);

б) функция вида (15)

$$
\omega\left[t\left(\tau_{0}\right)\right]=\frac{1}{\pi} \int_{0}^{2 \pi} \tilde{K}^{*}\left(\tau_{0} ; \tau\right) r\left(\tau_{0} ; \tau\right) \frac{v(\tau) d \tau}{\left|e^{i \tau}-e^{i \tau_{0}}\right|^{1-\beta}},
$$

где $r\left(\tau_{0} ; \tau\right)=\exp \left(-i \arg \left(e^{i \tau}-e^{i \tau_{0}}\right)\right)$, принадлежит классу $C_{\delta}$ для любой ограниченной функщии $v(\tau)$ и $\delta=\alpha-\beta$ (см. $\$ 51$ указанной работы).

Нетрудно убедиться в справедливости следующих неравенств [6]:

$$
\begin{aligned}
& \left\|\left[g^{\prime}(\tau)-g^{\prime}\left(\tau_{0}\right)\right]-\left[\tilde{g}^{\prime}(\tau)-\tilde{g}^{\prime}\left(\tau_{0}\right)\right]\right\|_{C_{\alpha}} \leq 2 \varepsilon ; \\
& \left\|\frac{e^{i \tau}-e^{i \tau_{0}}}{g(\tau)-g\left(\tau_{0}\right)} \frac{g^{\prime}(\tau)}{2 i e^{i \tau}}-\frac{e^{i \tau}-e^{i \tau_{0}}}{\tilde{g}(\tau)-\tilde{g}\left(\tau_{0}\right)} \frac{\tilde{g}^{\prime}(\tau)}{2 i e^{i \tau}}\right\|_{C_{\alpha}} \leq A_{1} \varepsilon ; \\
& \left\|\frac{g(\tau)-g\left(\tau_{0}\right)}{\bar{g}(\tau)-\bar{g}\left(\tau_{0}\right)}-\frac{\tilde{g}(\tau)-\tilde{g}\left(\tau_{0}\right)}{\tilde{g}(\tau)-\overline{\tilde{g}}\left(\tau_{0}\right)}\right\|_{C_{\alpha}} \leq A_{2} \varepsilon ; \\
& \left|\tilde{g}^{\prime}(\tau)\right| \geq \frac{[0,2 \pi]}{2}\left|g^{\prime}(\tau)\right| \\
& \left\|\left(g^{\prime}\left(\tau_{0}\right) \frac{\bar{g}(\tau)-\bar{g}\left(\tau_{0}\right)}{g(\tau)-g\left(\tau_{0}\right)}-\bar{g}^{\prime}\left(\tau_{0}\right)\right)-\left(\tilde{g}^{\prime}\left(\tau_{0}\right) \frac{\overline{\tilde{g}}(\tau)-\overline{\tilde{g}}\left(\tau_{0}\right)}{\tilde{g}(\tau)-\tilde{g}\left(\tau_{0}\right)}-\overline{\tilde{g}}^{\prime}\left(\tau_{0}\right)\right)\right\|_{C_{\alpha}} \leq A_{3} \varepsilon,
\end{aligned}
$$

где все содержащиеся в оценках константы не зависят от $\tilde{G}$. 
Учитывая (12), (13) и реэультат а) в (16), а также приведенные неравенства из [6] при малых значениях параметра $\varepsilon$, убеждаемся в справедливости неравенства

$$
\left\|\tilde{K}^{*}\left(\tau_{0} ; \tau\right)\right\|_{C_{\alpha-\beta}} \leq C_{1}(\beta) \varepsilon
$$

которое, в свою очередь, сог ласно результату б) из (15) приводит к оценке

$$
\|(H-\tilde{H}) v\|_{C_{\alpha-\beta}} \leq C_{0}(\beta)\|v\|_{C_{\alpha-\beta}} \varepsilon,
$$

где $C_{0}(\beta), C_{1}(\beta)$-абсолютные константы, а $v(\tau)$ - любая функция класса $C_{\alpha-\beta}$.

Предположим теперь, что $v(\tau)$ и $\bar{v}(\tau)$ - решения уравнений $(11)$ и $\left(11^{\prime}\right)$ соответственно. Тогда в силу (14), (14') имеем

$$
v-\tilde{v}=\tilde{A}^{-1}(H-\tilde{H}) v-\tilde{A}^{-1}\left(\tilde{f}_{0}-f_{0}\right) .
$$

Но согласно (16)

$$
\|A-\tilde{A}\|_{C_{\alpha-\beta}}=\sup _{\|v\|=1}\|(\tilde{A}-A) v\|_{C_{\alpha-\beta}} \leq \sup _{\|v\|=1} C_{0}(\beta)\|v\|_{C_{\alpha-\beta}}=C_{0}(\beta) \varepsilon
$$

и

$$
\left\|(\tilde{A}-A) A^{-1}\right\|_{C_{\alpha-\beta}} \leq\|\bar{A}-A\|_{C_{\alpha-\beta}}\left\|A^{-1}\right\|_{C_{\alpha-\beta}} \leq C_{0}(\beta)\left\|A^{-1}\right\|_{C_{\alpha-\beta}} \varepsilon .
$$

Если тепіерь предположить, что $\varepsilon<1 /\left(C_{0}(\beta)\left\|A^{-1}\right\|_{C_{\alpha-\beta}}\right)$, то обратный оператор $\bar{A}^{-1}$ будет равномерно (относительно $\bar{\Gamma}=\partial \bar{G}$ ) ограниченным в пространгстве $C_{\alpha-\beta}$.

$$
\left\|\tilde{A}^{-1}\right\|_{C_{\alpha-\beta}} \leq \frac{\left\|A^{-1}\right\|_{C_{\alpha-\beta}}}{1-\left\|(\tilde{H}-H) A^{-1}\right\|_{C_{\alpha-\beta}}} .
$$

Имея в виду еше и очевидную оценку для $\left\|f_{0}-\tilde{f}_{0}\right\|_{c_{\alpha-\beta}}$, нз (18) непосредственно получаем неравенство (10), справедлявое для всех $\varepsilon \leq \varepsilon_{0}$, где

$$
\varepsilon_{0}=\min \left\{\frac{\min |g(\tau)|}{2} ; \frac{\min \left|g^{\prime}(\tau)\right|}{2} ; \frac{1}{C_{0}(\beta)\left\|A^{-1}\right\|_{C_{\alpha-\beta}}}\right\},
$$

$A_{0}(\beta)$ - константа, зависящая только от $G$ и $\beta$ ( $\beta$ - побое положительное число, меньшее $\alpha$ ). Теорема доказана.

Оценка (10) позволяет с помощью функции $v(t)$, определенной заданием исходной области $G$, строить приближения к конформно отображающим функциям $w=\tilde{\varphi}_{\bar{v}}(z) \quad\left(\tilde{\varphi}_{\bar{v}}(0)=0, \tilde{\varphi}_{\bar{v}}^{\prime}(0)>0\right)$ (следуя [4]; см. формулу $\left.(7)\right)$ произвольной области $\tilde{G} \in G_{\varepsilon}$ на $|w|<1$.

Положим

$$
\bar{\varphi}_{v}(z)=z \exp \left(\frac{1}{\pi i} \int_{0}^{2 \pi} \frac{v(\tau) \bar{g}^{\prime}(\tau)}{\tilde{g}(\tau)-z} d \tau+\frac{i}{\pi} \int_{0}^{2 \pi} \frac{v(\tau) d|\bar{g}(\tau)|}{|\bar{g}(\tau)|}\right),
$$

где $v(\tau)$ - решение уравнения (9).

Если используем идею доказательства теоремы Племеля-Привалова [5] (§ 18) и неравенство (10), то установим справедливость следугоцен теоремы. 
Tеорема 2. Функция. $w=\tilde{\varphi}_{v}(z)\left(\tilde{\varphi}_{v}(0)=0, \tilde{\varphi}_{v}^{\prime}(0)>0\right)$, заданная в $\tilde{G}$ форхуулой (19), где $\tilde{G} \in G_{\varepsilon}, 0<\varepsilon<1$, в $\overrightarrow{\tilde{G}}$ допускает оценку

$$
\left|\tilde{\varphi}_{\bar{v}}(z)-\tilde{\varphi}_{v}(z)\right|<Q_{1} \varepsilon,
$$

где константа $Q_{1}$ зависит только от области $G$.

3. О квазиконформном отображении близких областей. Воспользуемся приемом выхода в плоскость основного гомеоморфизма $\tilde{W}(z)$. В плоскости $\tilde{W}(z)$ интегральные уравнения (9) и (9') принимают вид

$$
\begin{array}{ll}
\mu\left(\zeta_{0}\right)+\operatorname{Re} \frac{1}{\pi i} \int_{\tilde{W}(\Gamma)} \frac{\mu(\zeta) d \zeta}{\zeta-\zeta_{0}}=-\ln \left|\zeta_{0}-W_{0}\right| & \zeta_{0} \in \tilde{W}(\Gamma), \\
\mu\left(\zeta_{0}\right)+\operatorname{Re} \frac{1}{\pi i} \int_{\tilde{W}(\tilde{\Gamma})} \frac{\tilde{\mu}(\zeta) d \zeta}{\zeta-\zeta_{0}}=-\ln \left|\zeta_{0}-W_{0}\right| & \zeta_{0} \in \tilde{W}(\tilde{\Gamma}),
\end{array}
$$

где

$$
\begin{aligned}
\mu\left(\zeta_{0}\right) & =\nu\left[\tilde{W}^{-1}\left(g\left(\tau_{0}\right)\right)\right], \quad \tilde{\mu}\left(\zeta_{0}\right)=\tilde{v}\left[\tilde{W}^{-1}\left(\tilde{g}\left(\tau_{0}\right)\right)\right], \quad \tau_{0} \in[0,2 \pi], \\
W_{0} & =\tilde{W}(0), \quad \zeta=\tilde{W}[g(\tau)] \text { в }\left(20^{\prime}\right), \quad \zeta=\tilde{W}[\tilde{g}(\tau)] \text { в }\left(20^{\prime \prime}\right) .
\end{aligned}
$$

Таким образом, мы располагаем следуюшими данными [2]:

$\tilde{W}(\Gamma), \tilde{W}(\tilde{\Gamma}) \in C_{\gamma_{0}}^{1}[0,2 \pi], \quad \mu[\zeta(\tau)] \in C_{\gamma_{0}}[0,2 \pi], \quad \tilde{\mu}[\tilde{\zeta}(\tau)] \in C_{\gamma_{0}}[0,2 \pi]$.

В силу (8) имеем

$$
|\tilde{W}[g(\tau)]-\tilde{W}[\tilde{g}(\tau)]| \leq C_{l}\left(\tilde{W} ; G_{0}\right)|g(\tau)-\tilde{g}(\tau)| \leq C_{l}\left(\tilde{W} ; G_{0}\right) \varepsilon .
$$

Кроме того,

$$
\begin{gathered}
(\tilde{W}[g(\tau)])_{\tau}^{\prime}=\tilde{W}_{t}[g(\tau)] g^{\prime}(\tau)+\tilde{W}_{i}[g(\tau)] \bar{g}^{\prime}(\tau), \\
(\tilde{W}[\tilde{g}(\tau)])_{\tau}^{\prime}=\tilde{W}_{t}[\tilde{g}(\tau)] \tilde{g}^{\prime}(\tau)+\tilde{W}_{i}[\tilde{g}(\tau)] \overline{\bar{g}}^{\prime}(\tau), \\
(\tilde{W}[g(\tau)])_{\tau}^{\prime},(\tilde{W}[\tilde{g}(\tau)])_{\tau}^{\prime} \in C_{\gamma_{0}}[0,2 \pi] .
\end{gathered}
$$

Составим разность

$$
\begin{aligned}
& \left(\tilde{W}_{[g}[(\tau)]\right)_{\tau}^{\prime}-\left(\tilde{W}_{\tau}[\tilde{g}(\tau)]\right)_{\tau}^{\prime}=\tilde{W}_{t}[g(\tau)] g_{\tau}^{\prime}-\tilde{W}_{t}[g(\tau)] \tilde{g}_{\tau}^{\prime}+\tilde{W}_{t}[g(\tau)] \tilde{g}_{\tau}^{\prime}- \\
& -\tilde{W}_{t}[\tilde{g}(\tau)] \tilde{g}_{\tau}^{\prime}+\tilde{W}_{i}[g(\tau)] \bar{g}_{\tau}^{\prime}-\tilde{W}_{i}[g(\tau)] \overline{\bar{g}}_{\tau}^{\prime}+\tilde{W}_{\tilde{t}}[g(\tau)] \overline{\tilde{g}}_{\tau}^{\prime}-\tilde{W}_{i}[\tilde{g}(\tau)] \overline{\tilde{g}}_{\tau}^{\prime} .
\end{aligned}
$$

Учитывая в ней неравенства

$$
\begin{gathered}
\left.\left\|g^{\prime}(\tau)-\tilde{g}^{\prime}(\tau)\right\|_{C_{\gamma_{0}-\eta}} \leq \text { (const) }\right)_{1}\left\|g^{\prime}(\tau)-\tilde{g}^{\prime}(\tau)\right\|_{C_{\alpha}}, \\
\left.\left\|g^{\prime}(\tau)-\overline{\tilde{g}}^{\prime}(\tau)\right\|_{C_{\gamma_{0}-\eta}} \leq \text { (const) }\right)_{2}\left\|\bar{g}^{\prime}(\tau)-\overline{\bar{g}}^{\prime}(\tau)\right\|_{C_{\alpha},}, \\
\left.\left\|\tilde{W}_{t}[g(\tau)]-\tilde{W}_{t}\left[\tilde{g}^{\prime}(\tau)\right]\right\|_{C_{\gamma_{0}-\eta}} \leq \text { (const) }\right)_{3}|g(\tau)-\tilde{g}(\tau)|^{\eta}, \\
\left.\left\|\tilde{W}_{\hat{t}}[g(\tau)]-\tilde{W}_{\bar{t}}\left[\tilde{g}^{\prime}(\tau)\right]\right\|_{C_{\gamma_{0}-\eta}} \leq \text { (const) }\right)_{4}|g(\tau)-\tilde{g}(\tau)|^{\eta},
\end{gathered}
$$

где $0<\eta<\gamma_{0}$, в силу (8) получаем неравенство 


$$
\left\|(\tilde{W}[g(\tau)])_{\tau}^{\prime}-(\tilde{W}[\tilde{g}(\tau)])_{\tau}^{\prime}\right\|_{C_{\delta_{1}}} \leq C_{2}\left(\tilde{W} ; G_{0}\right) \varepsilon^{\eta},
$$

где $\delta_{1}=\gamma_{0}-\eta ; \eta-$ любое число между 0 и $\gamma_{0}$. Оптимальным вариантом в данном случае нам представляется $\eta=\gamma_{0} / 2$.

Таким образом, условия (8) в плоскости гомеоморфизма $\tilde{W}_{G_{0}}(z)$ заменяются условиями (21) и (22). Применяя теперь теорему 1 , убеждаемся в справедливости следующего предложения.

Теорема 3. Если области $G \subseteq G_{0} u \tilde{G} \in G_{\varepsilon}, \bar{G} \subset G_{0}$, границы которых

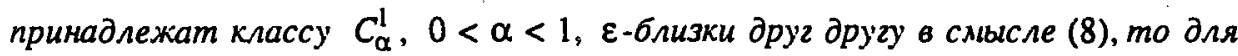
всех $\varepsilon \in\left(0 ; \varepsilon_{1}\right]$ справедлива оценка

$$
\|\mu(\zeta)-\tilde{\mu}(\zeta)\|_{C_{\gamma_{0}-\eta-\beta}} \leq C_{0}^{*}(\tilde{W} ; G ; \beta)\|\mu\|_{C_{\gamma_{0}-\eta-\beta}} \varepsilon^{\eta},
$$

где $\mu(\zeta)=v\left[\tilde{W}^{-1}(g(\tau))\right]$ и $\tilde{\mu}(\zeta)=\bar{v}\left[\tilde{W}^{-1}(\tilde{g}(\tau))\right]$ - единственные решения интегральньх уравнений $\left(20^{\prime}\right)$ и (20") соответственно, $0<\gamma_{0} \leq \min \{\alpha, \gamma\}, \beta$ - любое положительное число, леньшее $\gamma_{0}-\eta$, a $\eta \in\left(0, \gamma_{0}\right)$. Константа $C_{0}^{*}$ и льалое число $\varepsilon_{1}$ вполне определяются заданиель исходной области $G$ и гомеоліорфизліа $\bar{W}(z)$.

Теоремы 1 и 3 усиливают (в смысле порядка относительно $\varepsilon$ ) результаты, полученные в [7].

В заключение заметим, что если $G_{0}-$ круг, то можно указать конкретные виды коэффициента $q(z)$ уравнения (1), для которых гомеоморфизм $\tilde{W}(z)$ находится в явном виде. Это достигается с использованием схемы (2), (3), с учетом в ней формулы (4) и выражения $T_{G_{0}}\left(z^{n} \bar{z}^{m}\right)$ из $[1$, с. 44], где $n, m-$ неотрицательые целые числа.

1. Векуа Н. Н. Обобщелиые апалитические фупкцин. - М.: Физмапи из, 1953. -628 с.

2. Маножавидзе Г. Ф. Граничияа задача линейого соприжения со смещением и ее связъ с

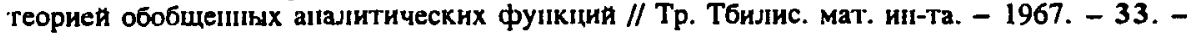
C. $82-87$.

3. Квеселива Д. А., Сиясопия З. В. О кцазихопформпом отображелии областей // Метрические вопросы теории фуікция. - Кнен: Наук. думка, 1980. -С. 53-65.

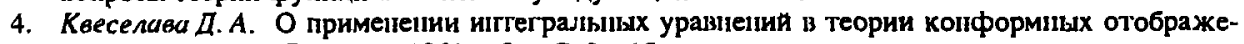
инй // Тр. ВЦ АН Грузин. - 1961. -2. - С. 3-15.

5. Мусхелишеили Н. Н. Сини уляріые иггтегралыне урапнения. - М.: Физматтиз, 1962. - 599 с.

6. Салссония 3. В. Колструктивцая реализация плоских квазикопформиых отображений, соответствуюцих линейым эллиптическим системам: Дис. ... ц-ра физ.-мат. иаук. - Тбилиси. $1991 .-247 \mathrm{c}$.

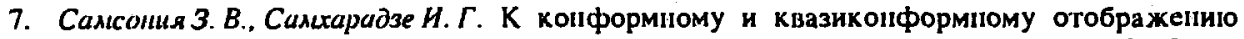
близких областей // Оптималыше метоцы нычислений и их применепие $\mathrm{k}$ обработке

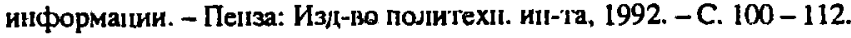

